\title{
High-level Features for Multimodal Deception Detection in Videos
}

\author{
*rodrigo.rill@\{inaoep.mx, hotmail.com $\}$ \\ 1. INAOE, Mexico \\ 2. CINVESTAV, Mexico \\ 3. UAT, Mexico
}

Rodrigo Rill-García ${ }^{*_{1}}$

Hugo Jair Escalante 1,2 , Luis Villaseñor-Pineda ${ }^{1}$ Verónica Reyes-Meza ${ }^{3}$

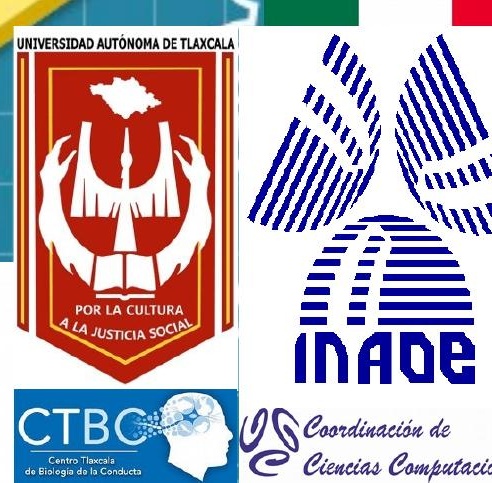




\section{Motivation}

- An "optimal" decision can be harmful if it is based on inaccurate (or wrong) data

- Purposely spreading inaccurate/wrong information is a way to mislead people

- Doing so for personal gain is the definition of deceiving 


\section{Problem Description}

- Deception detection is a hard task for humans

- Untrained people have an average accuracy $54 \%$ [1]

- Research supports that there is a difference in the way liars communicate in contrast with truth tellers

- Furthermore, such difference can be pointed out using Machine Learning 


\section{Problem Description (2)}

- There are many available sources of cues of deception interpretable by humans

- Eye movements

- Facial expressions

- Voice

- Speech

- Etc.

- Recent research suggests multimodal analysis can improve the performance of analyzing different modalities separately 


\section{Objective}

\section{To develop a multimodal}

information fusion method, inspired by classifier ensemble techniques, for deception detection in videos using high-level features 


\section{Related Work}

- "Detecting deceptive behavior via integration of discriminative features from multiple modalities" [2]

- Physiological features, thermal videos and transcriptions

- Early fusion

- Fused non-invasive features surpassed physiological ones

- "Deception detection using real-life trial data" [3]

- Videos (image) and transcriptions

- Early fusion

- Best performance with fused features

- "Deception detection in videos" [4]

$$
\begin{gathered}
\text { *No focus on } \\
\text { multimodal fusion } \\
\text { strategies }
\end{gathered}
$$

- Videos (image and audio) and trasncriptions

- Late fusion

- Best performance with fused features

- "Toward End-to-End Deception Detection in Videos" [5]

- Videos (image and audio)

- Early fusion

- Best performance with fused features 


\section{Datasets}

\begin{tabular}{|l|c|c|}
\hline Database & Court Trial & Abortion/Friend Spanish \\
\hline Deceptive/Truthful & $61 / 60$ & $22 / 21$ \\
\hline Subjects & 60 & 12 \\
\hline
\end{tabular}

Table 1. Summary of the databases used.
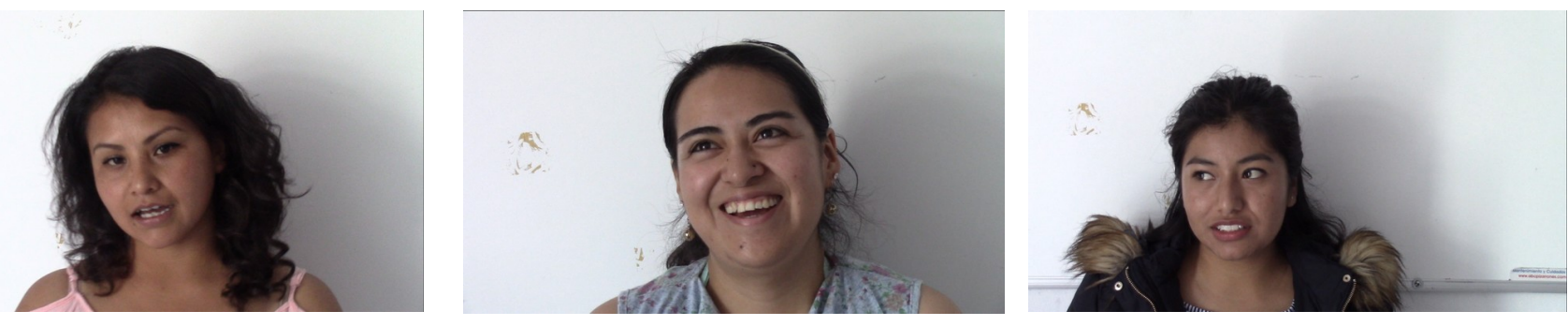

Figure 1. Examples of Spanish videos.
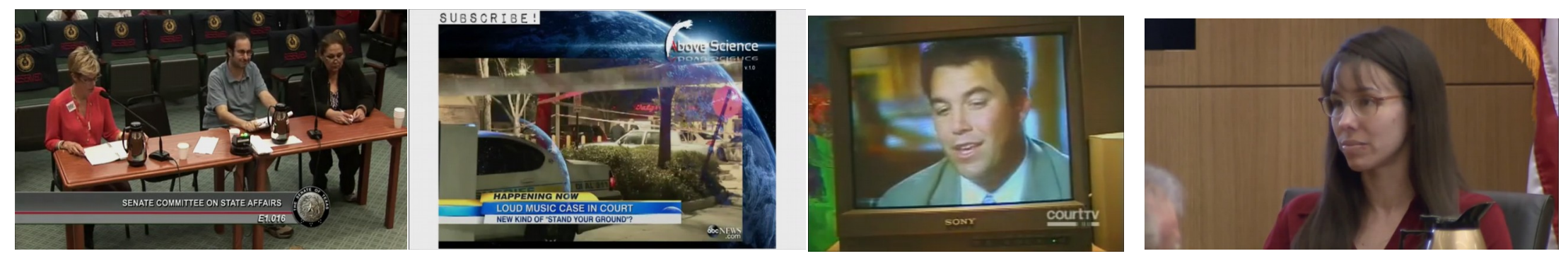

Figure 2. Examples of court videos [3]. 


\section{Feature Extraction}

\begin{tabular}{|c|c|c|c|}
\hline Modality & Visual & Accoustic & Textual \\
\hline \multirow{11}{*}{ Views } & $A U$ Int & Voice & Char 1-grams \\
\hline & AU Pres & Glottal Flow & Char 2-grams \\
\hline & Eye LM & MCEP & Char 3-grams \\
\hline & Facial LM & HMPDM & Char 4-grams \\
\hline & Gaze & HMPDD & POS 1-grams \\
\hline & \multirow[t]{6}{*}{ Head } & & POS 2-grams \\
\hline & & & POS 3-grams \\
\hline & & & POS 1-grams \\
\hline & & & BoW \\
\hline & & & LIWC \\
\hline & & & Syntax Info \\
\hline
\end{tabular}

Figure 3. The different views extracted for each of the 3 proposed modalities.

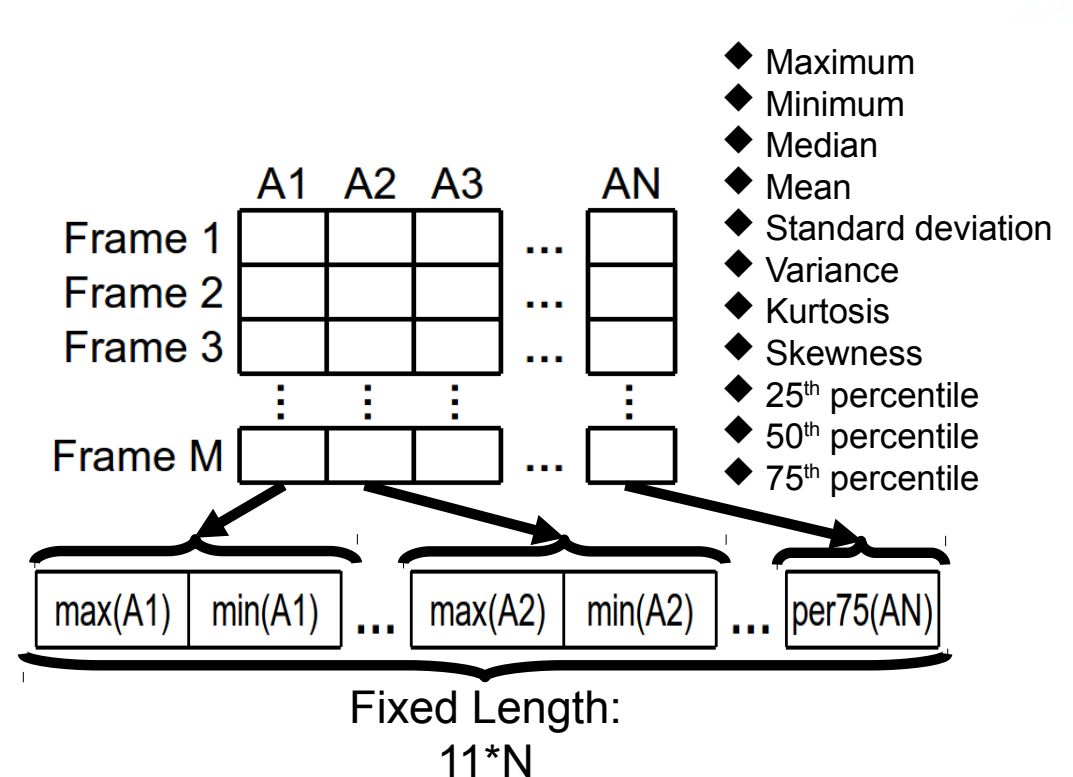

Figure 4. Creation of a fixed size vector from a numberof-frames-dependent matrix.

* OpenFace

** COVAREP

*** IBM Watson ASR, Google SyntaxNet, Python NLTK 


\section{Experimental settings}

- $\mathbf{N}$ feature sets (views) are extracted per video

- Textual modality is not extracted for Spanish

- Lack of a Mexican Spanish ASR system

- Metric: AUC ROC of the Deceptive class

- 10-folds cross-validation

- No subject seen in training is contained in the validation set 


\section{Single views}

- Court (Sklearn, LinearSVC)

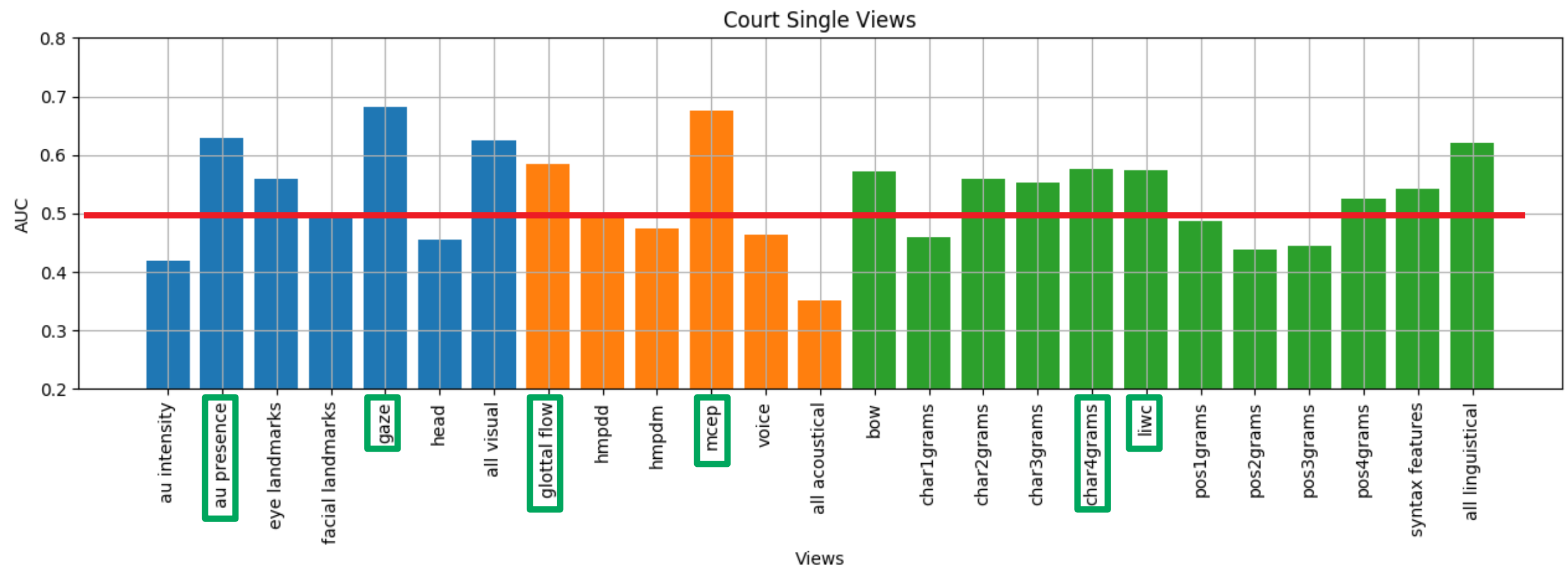

Figure 5. Results for single views/modalities in the court database. 


\section{Single Views (2)}

- Spanish (Sklearn, SVC: kernel=poly, C=0.01)

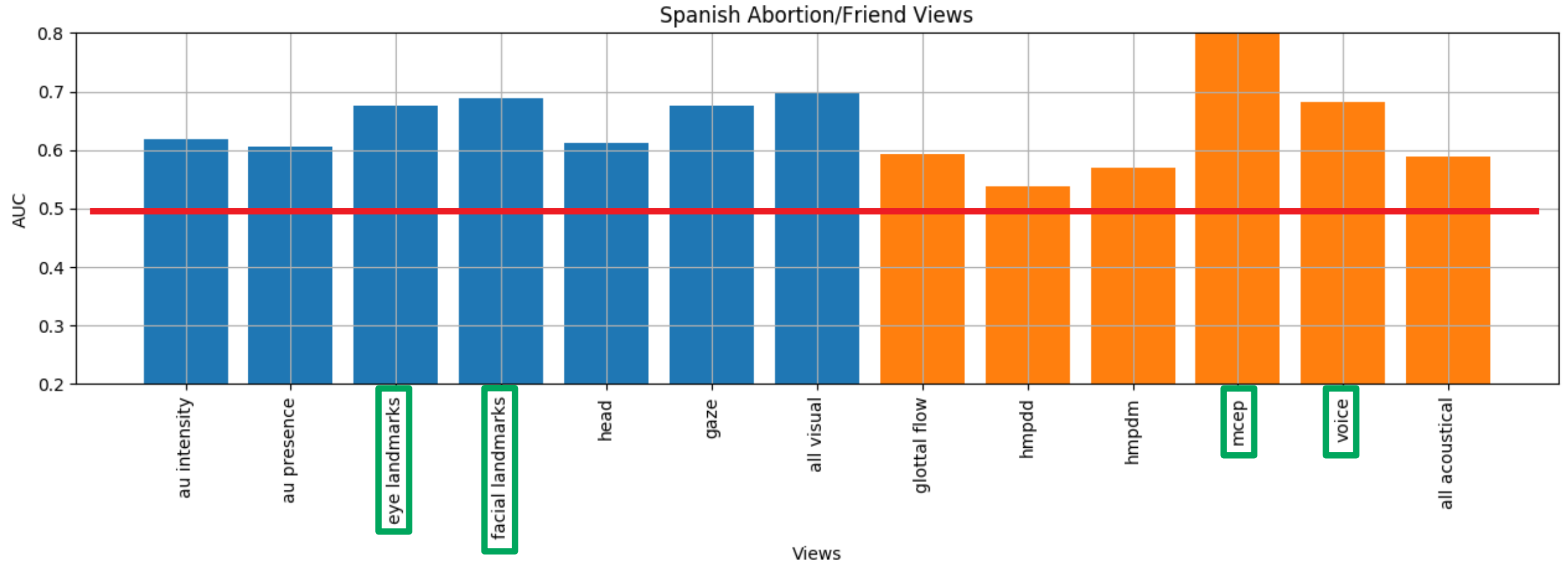

Figure 6. Results for single views/modalities in the Spanish database. 


\section{Complementarity}

Court 10-Subjects Cross Validation complementarity scores

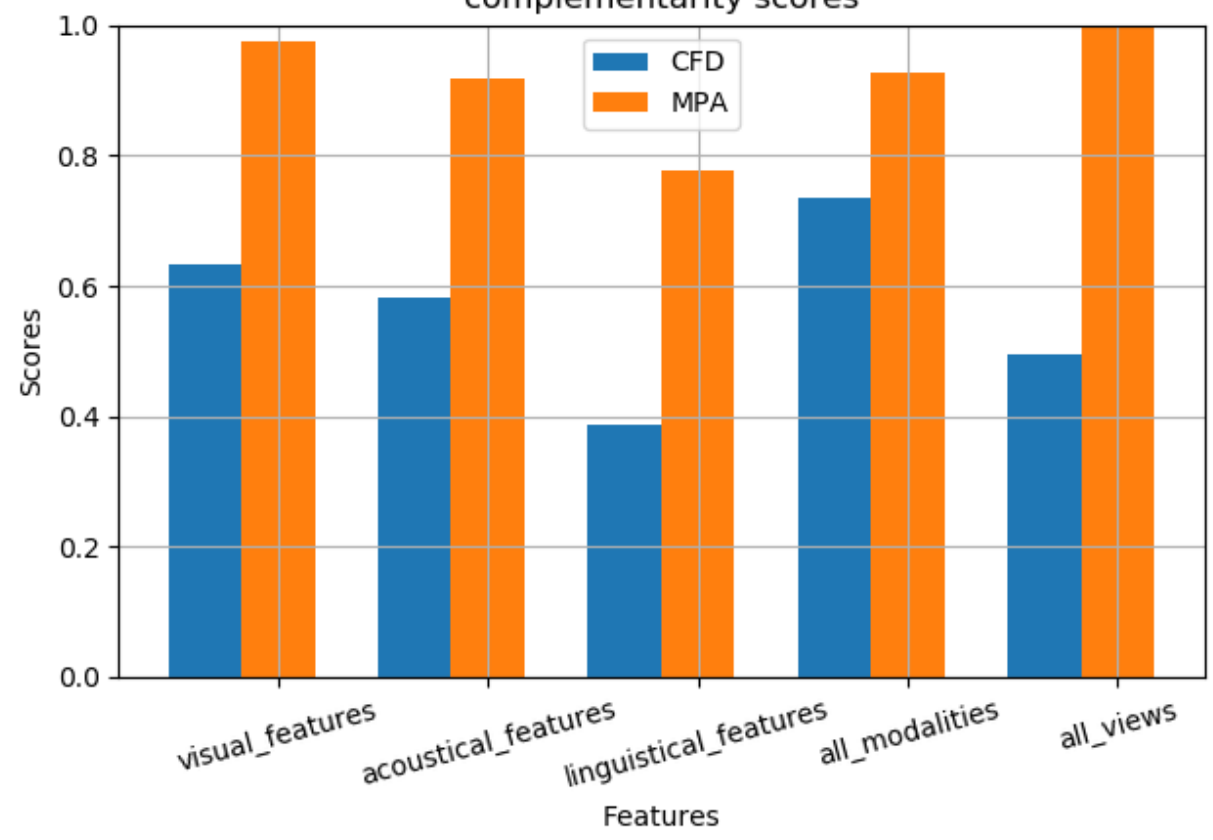

Figure 7. Complementarity measures for the court database.

The correct predictions from different views predict the whole datasets

\section{There is diversity in the errors} committed by each view

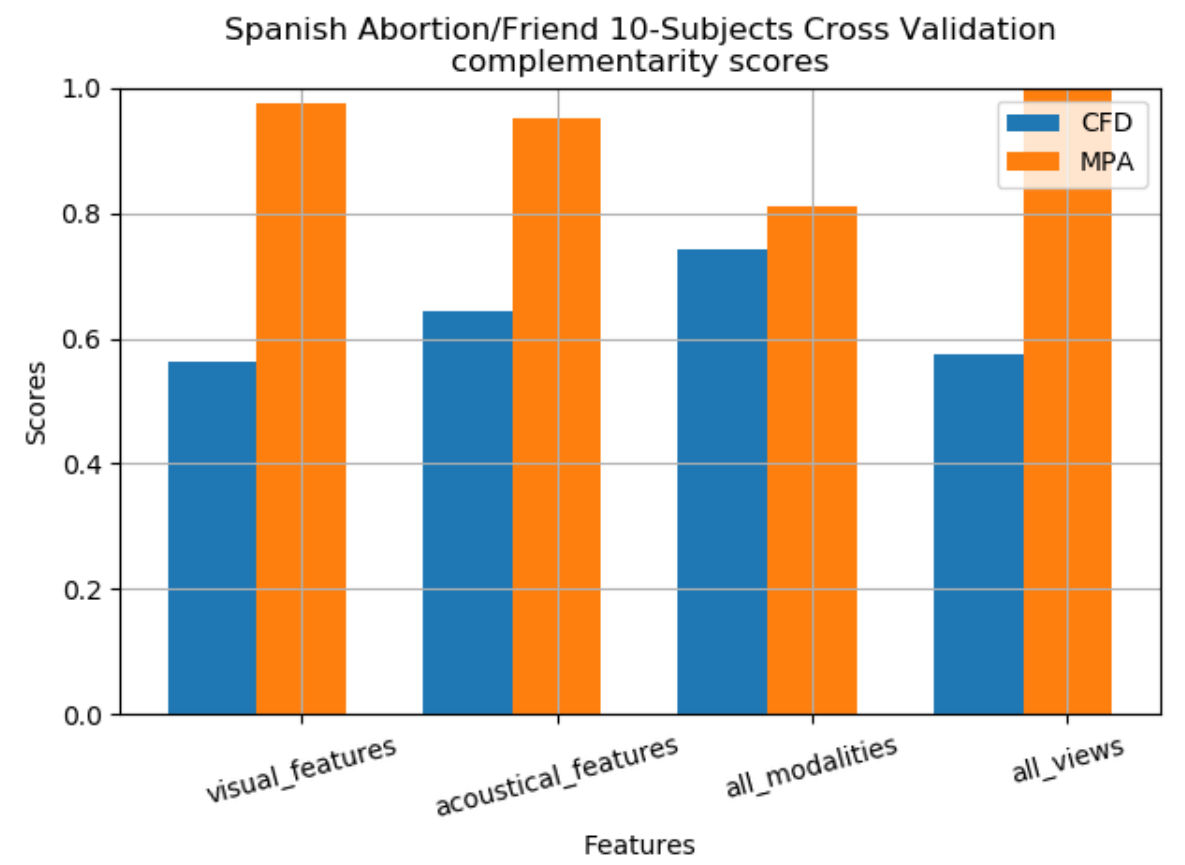

Figure 8. Complementarity measures for the Spanish database. 


\section{Proposed Methods (2)}

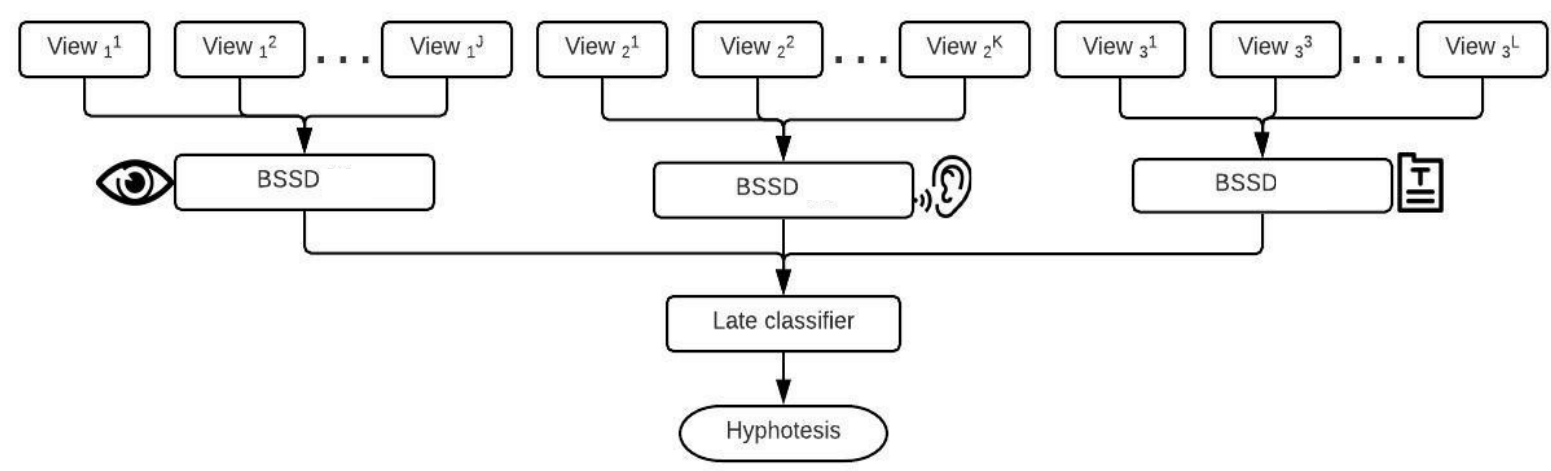

Figure 9. Block diagram of Hierarchical Boosting with Shared Sampling Distribution.

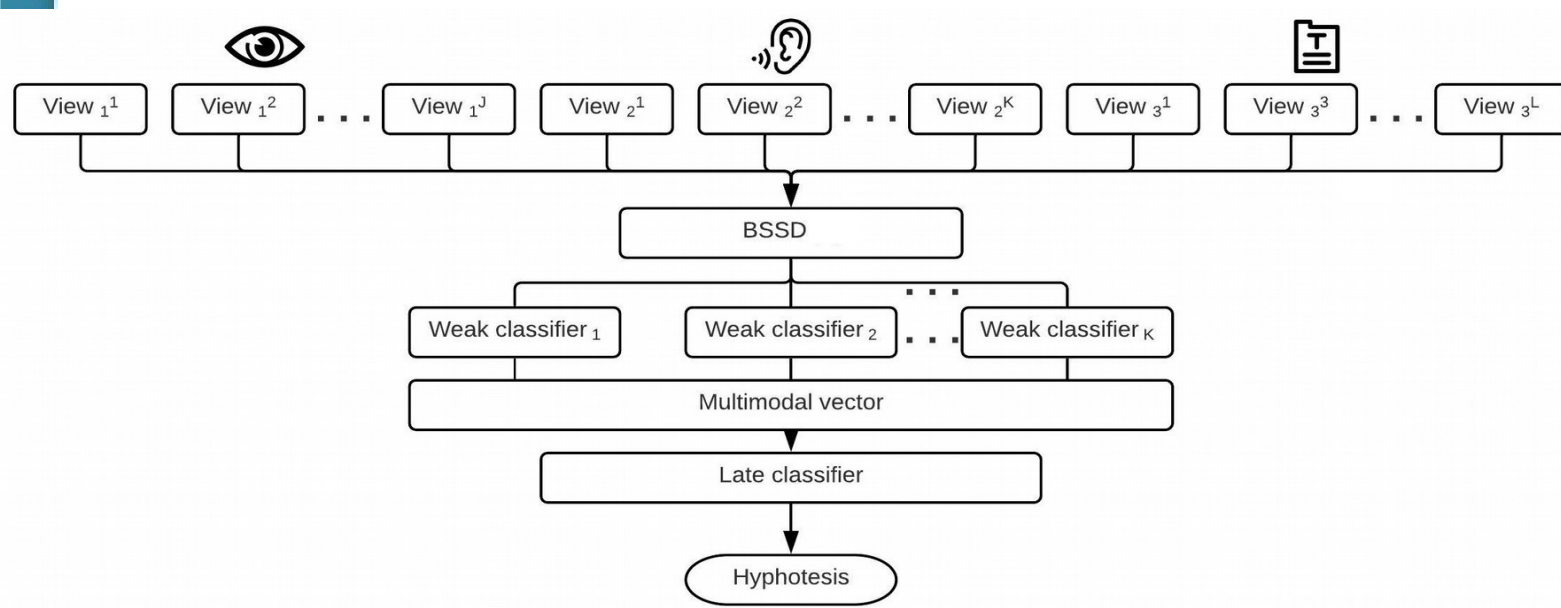

Figure 10. Block diagram of Stacked Boosting with Shared Sampling Distribution.
Algorithm 1: Boosting With Shared Sampling Distribution (BSSD) [5]

1. Input: $\left.z_{0}^{j}=\left\{x_{i}^{j}, y_{i}\right)\right\}_{i=1}^{n}, j=1, \cdots, M$.

2. Initialization: $W_{1}=\left\{w_{1}(i)=\frac{1}{n}\right\}_{i=1}^{n}$.

3. For $k=1$ to $k_{\max }$

(a) Sample $z_{k}^{j}$ from $z_{0}^{j}$ using the distribution $W_{k}$.

(b) Compute hypothesis $h_{k}^{j}$ from $z_{k}^{j}$ for each view $j$.

(c) Calculate error $\epsilon_{k}^{j}: \epsilon_{k}^{j}=P_{i \sim W_{k}}\left[h_{k}^{j}\left(x_{i}^{j}\right) \neq y_{i}\right]$

(d) If for each view: $\left\{\epsilon_{k}^{j}\right\}_{j=1}^{M} \leq 0.5$, select $h_{k}^{*}$ corresponding to $\epsilon_{k}^{*}=\min _{j}\left\{\epsilon_{k}^{j}\right\}$.

(e) Calculate $\alpha_{k}^{*}=\frac{1}{2} \ln \left(\frac{1-\epsilon_{k}^{*}}{\epsilon_{k}^{*}}\right)$.

(f) Update $w_{k+1}(i)=\frac{w_{k}(i)}{Z_{k}^{*}} \times e^{-h_{k}^{*}\left(x_{i}^{*}\right) y_{i} \alpha_{k}^{*}}$, where $Z_{k}^{*}$ is a normalizing factor.

4. Output: $F(x)=\sum_{k=1}^{k_{\max }} \alpha_{k}^{*} h_{k}^{*}\left(x^{*}\right)$.

5. Final hypothesis: $H(x)=\operatorname{sign}(F(x))$. 


\section{Fusion Results Court}
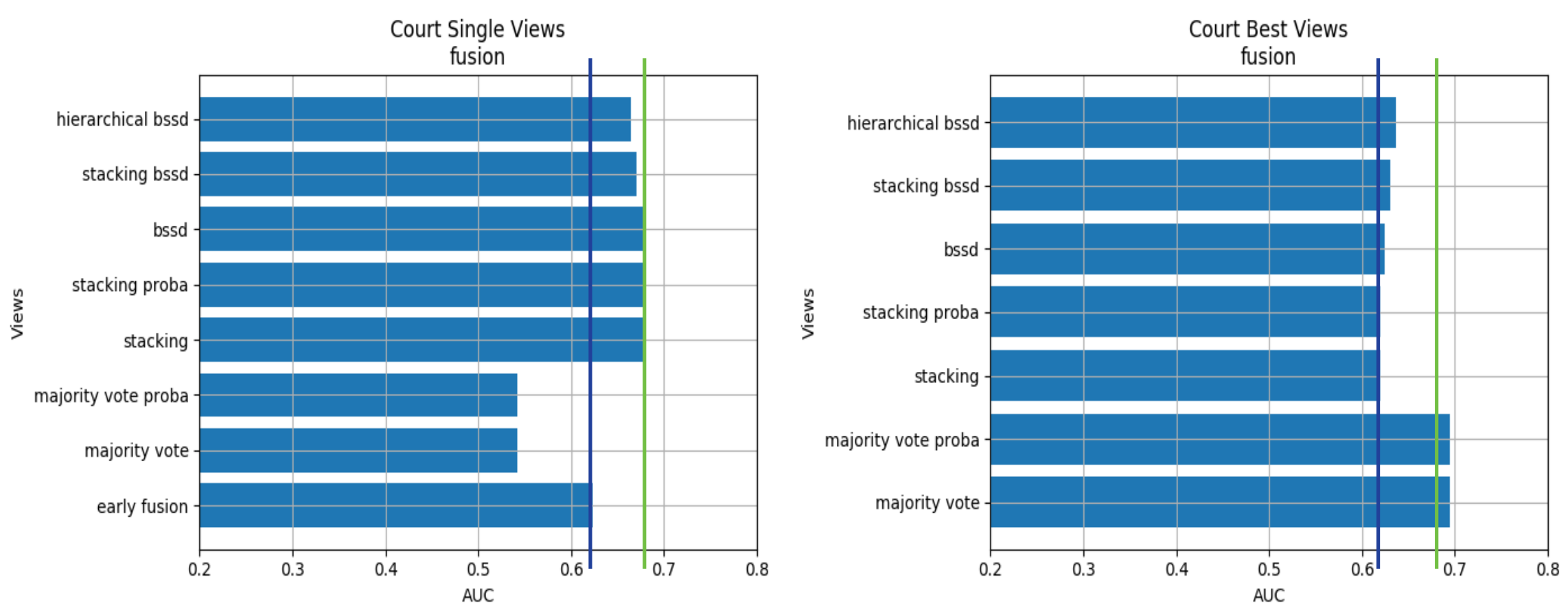

Figure 11. Results of fusion methods using all the views (left) and the best two views per modality (right) from the court database.

\section{Best view: Gaze direction $(0.683)$}

Early fusion: $\mathbf{0 . 6 2 3}$ 


\section{Fusion Results Spanish}
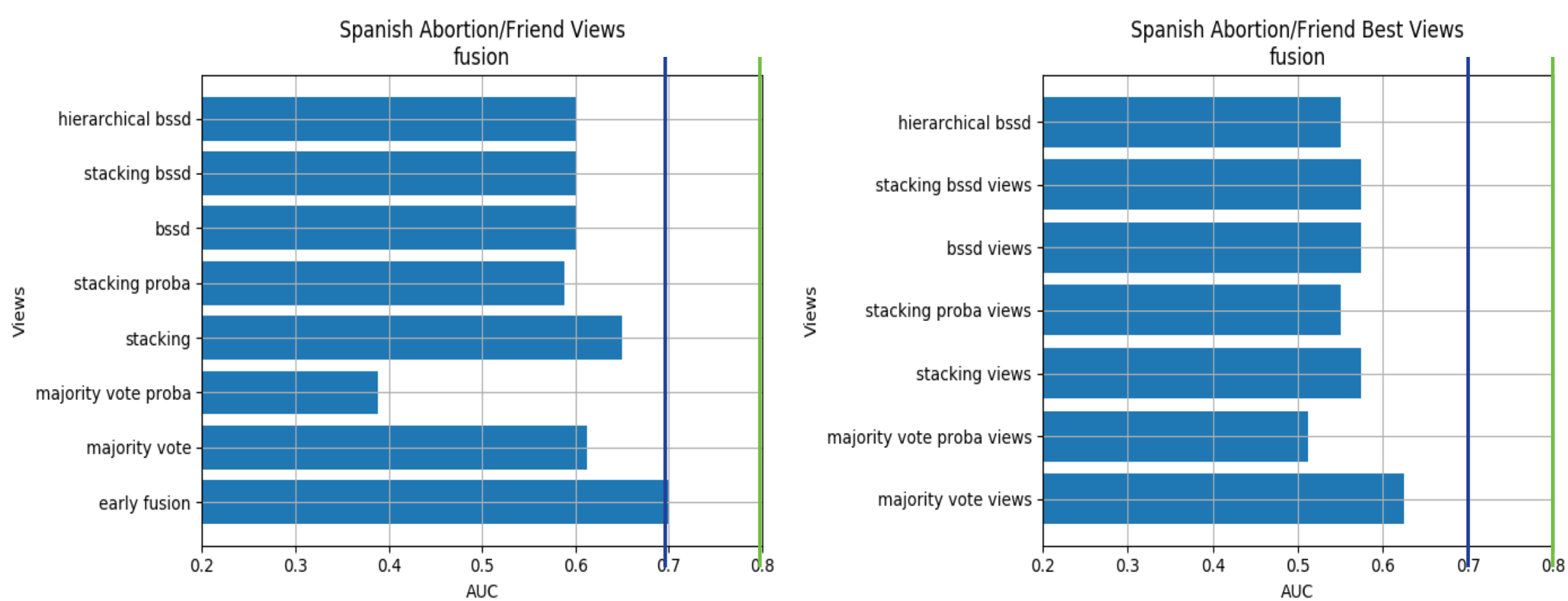

Figure 12. Results of fusion methods using all the views (left) and the best two views per modality (right) from the Spanish database.

Best view: MCPE $(0.856)$

Early fusion: 0.700 


\section{Conclusions}

- Despite language, context and topic differences, there are views useful for deception detection in both datasets

- Action units, eye landmarks, gaze direction (visual)

- MCEP, glottal flow (acoustical)

- Fundamental frequency and voiced/unvoiced intervals seem useful to detect deception on uninterrupted speech

- Complementarity analysis suggest it is useful to fuse features to improve performance

- Fusion is not trivial

- Alternatives to concatenating the multimodal features can improve the performance of a simple early fusion 


\section{Future work}

- To explore LSTM networks for temporal analysis of features

- To use boosting methods with tuned hyperparameters per view

- To study pure $N \boldsymbol{N}$ approaches preserving highlevel features

- To expand the Spanish dataset 


\section{References}

1.Bond Jr, Charles F and Bella M DePaulo (2006). "Accuracy of deception judgments". In: Personality and social psychology Review 10.3, pp. 214-234.

2.Abouelenien, Mohamed, Verónica Pérez-Rosas, Rada Mihalcea, et al. (2017). "Detecting deceptive behavior via integration of discriminative features from multiple modalities". In: IEEE Transactions on Information Forensics and Security 12.5, pp. 1042-1055.

3.Pérez-Rosas, Verónica et al. (2015). "Deception detection using real-life trial data". In: Proceedings of the 2015 ACM on International Conference on Multimodal Interaction. ACM, pp. 59-66.

4.Wu, Zhe et al. (2018). "Deception detection in videos". In: Thirty-Second AAAI Conference on Artificial Intelligence.

5.Barbu, Costin, Jing Peng, and Guna Seetharaman (2010). "Boosting information fusion". In: 2010 13th International Conference on Information Fusion. IEEE, pp. 1-8. 